\title{
An intrinsic source of reddening in the cataclysmic variable SS Cygni ${ }^{\star}$
}

\author{
S. Gaudenzi ${ }^{1}$, F. Giovannelli ${ }^{2}$, M. Mandalari ${ }^{1}$, M. Corradini ${ }^{1}$, and R. Lombardi ${ }^{1}$ \\ 1 Dpt. of Physics, Università La Sapienza, Roma Piazzale A.Moro 5, 00185 Roma, Italy \\ e-mail: franco.giovannelli@iasf-roma.inaf.it \\ 2 INAF - Istituto di Astrofisica Spaziale e Fisica Cosmica, Roma, Area di Ricerca CNR di Roma-2, via del Fosso del Cavaliere 100, \\ 00133 Roma, Italy
}

Received 26 November 2008 / Accepted 8 September 2010

\section{ABSTRACT}

\begin{abstract}
By using the INES archive of the IUE satellite, we analyzed the spectra of SS Cygni in quiescence in order to determine the value of the reddening of the binary system. The reddening of the system is reported in the literature with values that vary significantly from one another. The results demonstrate that the reddening is comprised by two components: the first, interstellar in origin, with a value $E(B-V)=0.04 \pm 0.02 \mathrm{mag}$, and the second, intrinsic to the binary system, with values up to $E(B-V)=0.16 \pm 0.02 \mathrm{mag}$. The intrinsic value of the reddening is variable along the quiescent phase and with orbital phase.
\end{abstract}

Key words. stars: dwarf novae - accretion, accretion disks - circumstellar matter - stars: individual: SS Cygni - ultraviolet: stars astronomical data bases: miscellaneous

\section{Introduction}

Cataclysmic variables (CVs) are binary systems in which the primary component is a white dwarf $\left(M_{\mathrm{wd}} \sim 1 M_{\odot}\right)$ and the secondary is a late-type main-sequence star $\left(M_{\mathrm{opt}}<1 M_{\odot}\right)$. Depending on the magnetic field intensity at the white dwarf, the accretion of matter from the secondary star onto the primary can occur either via an accretion disk or a channeling through the magnetic poles or in an intermediate way. Usually the mass transfer rate is ranging from $\dot{M} \sim 10^{-11}$ to $10^{-8} M_{\odot} \mathrm{yr}^{-1}$ (Patterson 1984; Smak 1985a). Cataclysmic variables have almost periodic flare-ups on a timescale of several weeks or years and with up to a few magnitudes in optical wavelengths; the duration of the outbursts is much shorter than the recurrence time.

A number of texts provide a comprehensive discussion of CV systems. Among them are Livio \& Shaviv (1984), Hack \& La Dous (1990, 1993), Warner (1995), Hellier (2001). Several older reviews also provide an in-depth discussion of CVs, including La Dous (1994) and Patterson (1994). For a more contemporary discussion, please see the recent review paper by Giovannelli (2008). In addition, we recommend the important reviews about the evolution of CVs and low mass X-ray binaries (LMXBs) by Patterson $(1984,1998)$ and Ritter (2008).

Historically, the classification of CVs was based on the optical outburst properties, by which one may distinguish four groups of CVs: (i) classical novae; (ii) recurrent novae; (iii) dwarf novae; (iv) nova-like objects (see, e.g., Robinson 1976; Cordova \& Mason 1983). In this scheme of classification, SS Cyg $\left(\alpha_{2000}=21^{\mathrm{h}} 42^{\mathrm{m}} 48^{\mathrm{s}} .2 ; \delta_{2000}=+43^{\circ} 35^{\prime} 09^{\prime \prime} .88\right.$ with the galactic coordinates $\left.l_{2000}=090.5592, b_{2000}=-07.1106\right)$, whose distance is $166.2 \pm 12.7 \mathrm{pc}$ (Harrison et al. 1999), is a dwarf nova and of those the brightest one. Its optical magnitude ranges from $\sim 12$ to $\sim 8.5$ during quiescent and outburst phases, respectively.

\footnotetext{
* Based on IUE measurements, available in the INES archive.
}

Because of these characteristics, it is the most observed CV, not only in the optical, where measurements are available from the end of the 19th century (see, e.g., Mattei et al. 1985, 1991) to the present, but also in other wavelength regions. However, the former classification is neither self-consistent nor adequate, and it is much better to consider the observed accretion behavior as a primary litmus (Smak 1985b). One obvious advantage of such an approach is connected with the time scales of various accretion phenomena, which are sufficiently short, so as to avoid any major observational bias: the mass accretion rates in CVs, as we already said, range from $\sim 10^{-11}$ to $10^{-8} M_{\odot} \mathrm{yr}^{-1}$; the time scales are from tens of seconds (oscillations in dwarf novae at outbursts) to years (for example, the super-outbursts of SU UMa stars or the long-term variations in VY Scl stars). SS Cygni shows oscillations of $\sim 10 \mathrm{~s}$ both in optical and in X-ray ranges, orbital modulations $\left(P_{\text {orb }} \simeq 6.6 \mathrm{~h}\right.$ ) of the intensities of Balmer and UV emission lines and of the continuum, and almost periodic outbursts $\left(P_{\text {outb }} \sim 50\right.$ days, Howarth 1978). All these characteristics together with the relative high luminosity both in outburst and in quiescence render SS Cygni the most appropriate laboratory for studying the physical processes occurring in dwarf novae and in CVs in general (see the review by Giovannelli \& Martinez-Pais 1991).

However, despite the enormous amount of multifrequency experimental data spread over many years, the morphology and the nature of SS Cygni are still unsettled questions. Indeed, SS Cygni was classified as a non-magnetic CV (NMCV) by Bath \& van Paradijs (1983). Ricketts et al. (1979) explained the $\mathrm{X}$-ray emission from SS Cygni as owing to the radial inflow of matter onto a magnetized white dwarf $\left(B \sim 10^{6} \mathrm{G}\right)$ from a disrupted accretion disk. Fabbiano et al. (1981), using optical-UV spectroscopy of SS Cygni, noted that its behavior is not compatible with a viscous disk model and confirmed the magnetic nature of the white dwarf with $B \leq 1.9 \times 10^{6} \mathrm{G}$, by using coordinated X-ray measurements. Further multifrequency data of SS Cygni showed the incompatibility of its behavior with that 
of NMCV, and strongly favored its classification as that of an intermediate polar (see, e.g. Giovannelli et al. 1985; Giovannelli \& Martinez-Pais 1991; Kjurkchieva et al. 1998; Marchev et al. 1999; Gaudenzi et al. 2002; Long et al. 2005; Schreiber et al. 2003; Schreiber \& Lasota 2007).

Moreover, in SS Cyg, $L_{\mathrm{hard}-\mathrm{X}}<L_{\mathrm{UV}+\text { soft-X}}$. This is compatible with thermonuclear burning onto the WD surface. Thermonuclear burning was first suggested by Mitrofanov (1978). Gaudenzi et al. (2002) found that thermonuclear burning can occur in $\sim 24 \%$ of the WDs surface. Recently, Kording et al. (2008) detected a radio jet from SS Cyg. The hardness intensity diagram shows an analogy between X-ray binaries (XRBs) and SS Cyg. Upper limits to linear and circular polarizations have been found as $3.2 \pm 2.7 \%$ and $-3.2 \pm 2.7 \%$, respectively.

INTEGRAL/IBIS and SWIFT/XRT observations have shown that a conspicuous number of CVs have a strong hard $\mathrm{X}$-ray emission (Landi et al. 2009). In their published sample of $22 \mathrm{CVs}, 21$ are classified as magnetic CVs (MCVs) (intermediate polar: IP) and only one (SS Cyg) as NMCV, meanwhile all its characteristics are practically equal to those of the other 21 objects. This is one more strong circumstantial proof in favor of the magnetic nature of SS Cyg. Scaringi et al. (2010) reported the detection of one more IP: AO Psc, which is added to the former sample.

However, in the literature there are many papers that seem to contradict the intermediate polar nature of SS Cyg. Indeed, Gnedin et al. (1995) found from observations of intrinsic circular polarization in SS Cyg performed in the wings of the Balmer hydrogen lines that the true value of the magnetic field probably lies in the range $0.03<B<0.3 \mathrm{MG}$.

Mauche (1996) detected by using Extreme Ultraviolet Explorer satellite observations quasi-periodic oscillations (QPOs) from SS Cyg with a period in the range 7.19-9.3 s. This variation correlates with the extreme ultra-violet (EUV) flux as $P \propto I_{\mathrm{EUV}}^{-0.094}$. With a magnetospheric model to reproduce this variation, he found that a high-order, multipole field is required, and that the field strength at the surface of the white dwarf is $0.1<B<1 \mathrm{MG}$. This field strength is at the lower extreme of those measured or inferred for bona fide magnetic cataclysmic variables. However, they do not exclude the possibility that at an outburst the accretion of matter could occur onto the magnetic poles of the white dwarf.

With regard to this question, Mauche et al. (1997) discussed the case of UV line ratios of $\mathrm{CVs}$, which seem to be almost independent of the nature (magnetic or not) of CVs. Okada et al. (2008) found from CHANDRA HETG (High Energy Transmission Grating) observations in SS Cyg that the spectrum in quiescence is dominated by $\mathrm{H}$-like $\mathrm{K}_{\alpha}$ lines, and is dominated in outburst by He-like lines, which are as intense as $\mathrm{H}$-like lines. The broad line widths and line profiles indicate that the lineemitting plasma is associated with the Keplerian disk. In quiescence the lines are narrower and are emitted from an ionizing plasma at the entrance of the boundary layer. Ishida et al. (2009) found from SUZAKU observations of SS Cyg that the plasma temperature in quiescence is $20.4 \mathrm{keV}$ and in outburst $6.0 \mathrm{keV}$. The $6.4 \mathrm{keV}$ line is resolved in narrow and broad components, which indicates that both the white dwarf and accretion disk contribute to the reflection. The standard optically thin boundary layer is the most plausible picture of the plasma configuration in quiescence. The reflection in outburst originates from the accretion disk and an equatorial accretion belt. The broad 6,4 keV line suggests that the optically thin thermal plasma is distributed on the accretion disk, in a manner similar to that of a solar corona.
Long et al. (2005) found by fitting the double-peaked line profile in SS Cyg that the FUV line-forming region is concentrated closer to the white dwarf than the region that forms the optical lines. Their study provides no evidence of a hole in the inner disk.

The system SS Cygni is also important as laboratory for the study of circumstellar dust in CVs. Indeed, Jameson et al. (1987) detected IR emission from SS Cygni in outburst in the IRAS Bands I and II $(11.8 \mu \mathrm{m}$ and $24.4 \mu \mathrm{m})$. The most likely origin of the IR emission is circumstellar dust heated by the enhanced UV flux during outburst. Dubus et al. (2004) performed optical and mid-IR observations of several CVs including SS Cygni in quiescence. For SS Cygni the measurements at $11.8 \mu \mathrm{m}$ are consistent with the upper limits obtained by Jameson et al. (1987) when the source was not yet in full outburst. The observed variability in the mid-IR flux on short time scales is hardly reconcilable with intrinsic or reprocessed emission from circumbinary disk material, while on the contrary a free-free emission from a wind should be. If any sizeable circum-binary disk is present in the system, it must be self-shadowed or perhaps dust free, with the peak thermal emission shifted to far-IR wavelengths.

The orbital parameters of the binary system were derived by Giovannelli et al. (1983) by using theoretical and experimental constraints from measurements obtained in different energy regions. They are $i=40_{-2}^{\circ+1}, M_{1}=0.97_{-0.05}^{+0.14} M_{\odot}$, $M_{2}=0.56_{-0.03}^{+0.08} M_{\odot}, R_{2}=0.68_{-0.01}^{+0.03} R_{\odot}, R_{\text {od }}=2.9 \times 10^{10} \mathrm{~cm}$, $R_{\mathrm{id}}=3.6 \times 10^{9} \mathrm{~cm}$, where 1 and 2 refers to the primary and secondary star, respectively. $R_{\mathrm{od}}$ and $R_{\mathrm{id}}$ are the outer and inner accretion disk radius. These parameters have been confirmed by direct measurements of radial velocities (Martinez-Pais et al. 1994). Martinez-Pais et al. determined also that the optical companion of SS Cygni system is a K2-K3 late-type star.

The galactic reddening in the direction of SS Cyg is 0.512 mag (NED catalog; Schlegel et al. 1998), but it is irrelevant for SS Cyg and nearby objects because they are a few hundreds of parsecs distant.

The determination of $E(B-V)$ has been always very difficult and the results obtained in the literature range from $0.04 \pm$ 0.03 mag (Verbunt 1987; La Dous 1991), to 0.07 mag (Bruch $\&$ Engel 1994), to $0.08 \pm 0.08 \mathrm{mag}$ (Holm \& Gallagher 1974), to $0.11 \pm 0.02 \mathrm{mag}$ (Giovannelli et al. 1984), which is an average value determined by using two dozens of spectra available at that time. The latter value completely agrees with that determined by Zuckerman (1961). Kiplinger (1979) determined a value $E(B-V)=0.13 \pm 0.03$ mag from his optical spectrophotometric observations during a quiescent phase, integrated with UV (Wu 1976) and IR data (Szkody 1977).

Wallerstein (1963) briefly discussed the absolute magnitude and reddening of SS Cyg. He performed a comparison of the strengths of the interstellar lines in SS Cyg with those in the spectra of stars in the same part of the sky, and concluded that the reddening of the stars in that region is small $(0.0 \leq E(B-$ $V) \leq 0.06 \mathrm{mag}$ ) and that it is likely that SS Cyg is unreddened.

A natural question arises from this. Why are the values of $E(B-V)$ so different? Could a physical reason exist for these differences? We suggest that it is possible that the reddening depends both on a constant, interstellar component, and on another element intrinsic to the binary system. It is this element which is variable. The test of this hypothesis is the principal reason for this paper.

We have therefore re-analyzed all the IUE spectra of SS Cygni in order to investigate the behavior of the reddening. 
We present here the results of the analysis of the UV spectra of SS Cygni in quiescence with data from the IUE archive. We used all short wavelength (SW) and long wavelength (LW) spectra for which the short-long wavelength junction was possible.

Our results show a variable reddening of the system when the quiescent phase is developing.

\section{Journal of observations}

Table 1 reports the journal of observations that were performed with the IUE observatory by several groups including our own. The data are now available in the INES archive.

Columns report 1) progressive numbers; 2) the image number; 3) the heliocentric Julian day (HJD); 4) the orbital phase computed using the ephemeris of Feldt \& Chincarini (1980); 5) the exposure time $t_{\text {exp }}$; 6) $t \%$ which is the time elapsed since the end of the preceding outburst expressed in percent of the duration of the quiescent phase; 7$)$ number $\left(n_{x}, x=a, b, c\right)$ corresponding to the quiescence during which spectra were obtained (see Fig. 1).

\section{Data analysis}

All quiescent spectra acquired with the large slit have been considered for this analysis. Information on the optical state of SS Cygni was provided by the American Association of Variable Stars Observers (AAVSO). The light curves for the periods of interest are shown in Fig. 1.

In order to evaluate the initial $t_{\mathrm{i}}$ and final $t_{\mathrm{f}}$ times of a quiescence by utilizing the data from the light curves, we performed a linear fit for

1. the declining phase data of the preceding outburst;

2. the quiescence phase data;

3. the arising data of the following outburst.

We derived $t_{\mathrm{i}}$ and $t_{\mathrm{f}}$ by determining the two intersection points between the first and second linear fit $t_{\mathrm{i}}$ and between the second and the third one $t_{\mathrm{f}}$. The quiescence length is then given by

$t_{\mathrm{q}}=t_{\mathrm{f}}-t_{\mathrm{i}}$.

For the scope of this work, it is helpful to utilize the time elapsed from the end of the preceding outburst as percentage of the whole quiescence period:

$t_{\%}=\frac{\left(\mathrm{HJD}-t_{\mathrm{i}}\right) \cdot 100}{t_{\mathrm{q}}}$.

\section{Results}

In order to evaluate the reddening of the system we used the whole INES archive spectra for which a short-long wavelength junction was possible. The name of each pair is formed by longshort (LS) and four digits: the first two and the last two refer to the last digits of the long wavelength (LW) and short wavelength (SW) spectra, respectively.

Table 2 reports these pairs of spectra together with the results (see below).

Two independent methods were used to determine the value of the reddening $E(B-V)$ by filling the $2175 \AA$ absorption bump (Seaton 1979; Fitzpatrick \& Massa 1986, 1988):

1. after the determination of the continuum from 1250 to $3250 \AA$, we applied nine successive different values of $E(B-$ $V$ ), from 0.04 to $0.20 \mathrm{mag}$ with steps of $0.02 \mathrm{mag}$ to find the value corresponding to the best de-bumping;
2. in the second case an automatic procedure was used to trace a continuum between $1795 \AA$ and $3080 \AA$ and to evaluate the root mean square (rms) between the intensity values of the absorption feature (1970-2500 $\AA$ ) and the corresponding continuum values: the $E(B-V)$ value minimizing the rms has been chosen for the reddening evaluation.

A comparison between the results obtained by the two methods allowed us to solve the few remaining uncertain cases and to refine the determination of the uncertainty to be associated to $E(B-V)$. These results are reported in Table 2 and are shown in Figs. 2 to 6 . It is evident that $E(B-V)$ shows different values varying $t \%$ and orbital phase $(\Phi)$.

Figure 2 shows $E(B-V)$ vs. $t \%$ for a quiescent phase between a short outburst $(S)$ and a long outburst $(L)$ :

- at the beginning of the quiescence we find the minimum value for the correction, that is $0.04 \mathrm{mag}$;

- during the quiescence, the $E(B-V)$ values range from the minimum of $0.04 \mathrm{mag}$ to a maximum of $0.16 \mathrm{mag}$. The higher values are roughly concentrated between $t \% \sim 30$ and $t_{\%} \sim 70$.

Unfortunately, not all regions of quiescence are covered by experimental data.

Figure 3 shows that from about the half and the end of a quiescence between a long or long(anomalous) outburst ( $L$ or $L(A))$ and a short outburst $(S), E(B-V)$ decays from a maximum of $\sim 0.16$ mag to a minimum of $\sim 0.06$ mag. Unfortunately data in the first half of the quiescence are not available. However, collecting the available data all together, independently of the kind of quiescence, as shown in Fig. 4, an increasing of the reddening in the first half of the quiescence from the minimum of $\sim 0.04$ mag to the maximum of $\sim 0.16 \mathrm{mag}$ is evident, with the exception of two data. In the second half of the quiescence, $E(B-V)$ decreases until the minimum value.

Figures 5 and 6 show the $E(B-V)$ behavior as a function of the orbital phase. Figure 6 shows evidence of the presence of a modulation of the data following a long and/or long anomalous outburst, while no definite conclusion can be inferred concerning the phase-dependent behavior after a short outburst, as shown in Fig. 5.

Figure 7 shows the contour of $E(B-V)$ in the $\Phi-t \%$ plane. Evidently the maximum value of $E(B-V)=0.16$ mag is at about $70 \leq t \% \leq 80$ and $0.5 \leq \Phi \leq 0.6$. However, it is important to note that the enhancement of $E(B-V)$ starts at $\Phi \sim 0.2$, where its value is the minimum (0.04 mag), growing until its maximum and then decreasing again before ending at $\Phi \sim 0.9$, where its value is again the minimum. The growing of $E(B-V)$, along the quiescent phase starts at about $t \% \sim 30$, reaching the maximum at about 70-80 and then decreasing again until the next outburst. In other words, soon after the end of an outburst, the reddening is at the minimum value. This indicates that no opaque material is present in the system, and that this material forms during the days following the outburst.

We have performed a $t$-student hypothesis test on the $E(B-$ $V)$ values in order to check the statistical significance of their variations. The selected hypothesis is the following: $\overline{E(B-V)}>$ $0.04 \mathrm{mag}$, this value being the minimum obtained from our data and also reported once in the literature (Verbunt 1987). The test indicates that the probability for having values $E(B-V)>$ $0.04 \mathrm{mag}$ is $99.95 \%$. This means that the values higher than the minimum (0.04 mag) are caused by an additional variable component intrinsic to the system itself. 

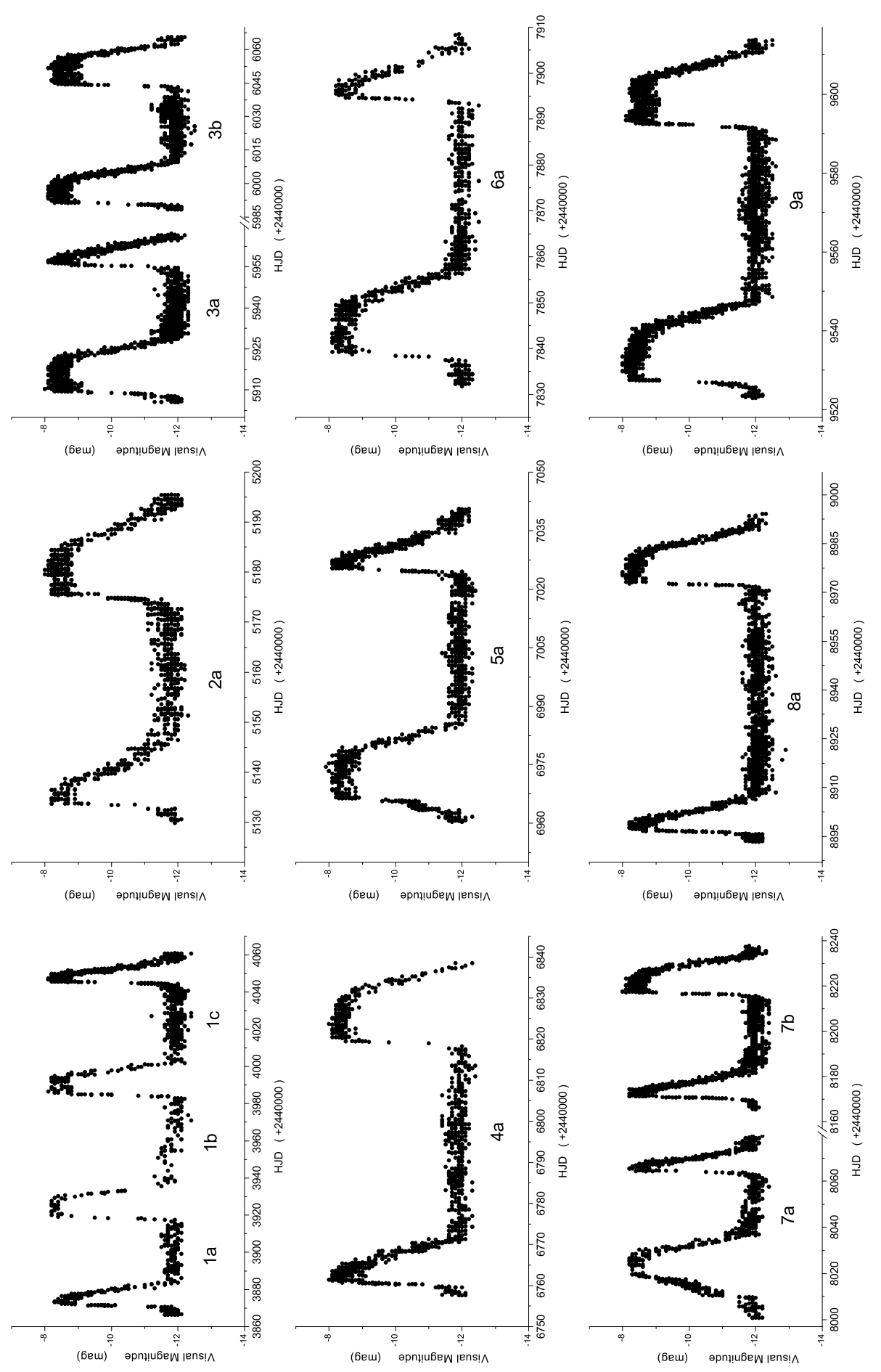

Fig. 1. Optical light curves of SS Cygni in the epochs of the IUE measurements used in this paper (Mattei et al. 1985, 1991).

\section{Discussion}

\subsection{Fullerenes}

It is reasonable to ask what sort of candidate material we hypothesize for this additional variable component, intrinsic to the system itself, in the $2175 \AA$ A region.

As shown by Iglesias-Groth (2004) fullerenes and buckyonions are the best candidates to explain the shape, width and peak energy of the most prominent feature of the UV $2175 \AA$ bump. Concerning the diffuse interstellar medium (ISM), the positions and widths observed for the $2175 \AA$ bump can be explained by the power-law-size distribution proposed by Iglesias-Groth (2004). In her calculations of the density of fullerenes and buckyonions, she uses a comparison between the theoretical cross-sections of her model and the UV bump data. In addition, some studies are available in the literature confirming the formation of fullerenes and buckyonions in the laboratory 


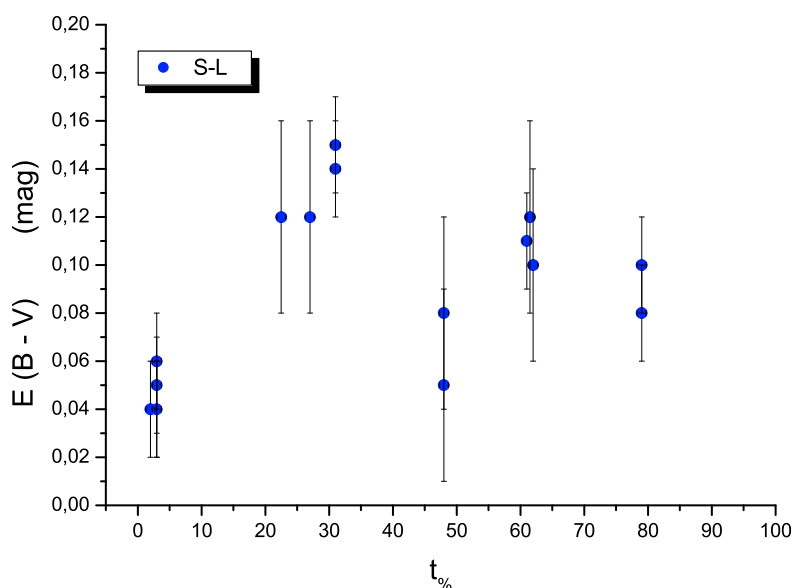

Fig. 2. $E(B-V)$ values versus $t_{\%}$ for spectra belonging to a quiescence between a short and a long outburst.

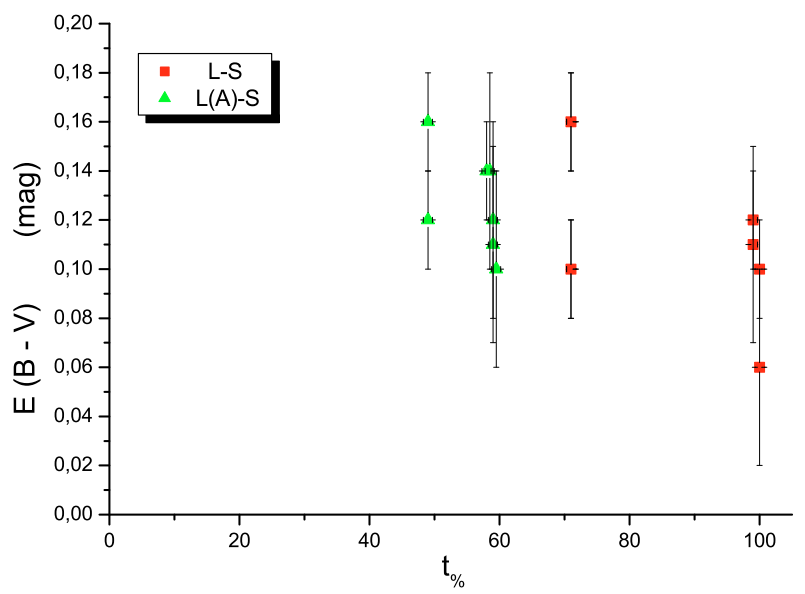

Fig. 3. $E(B-V)$ values versus $t \%$ for spectra belonging to a quiescence between a long/long(anomalous) and a short outburst.

(Kuznetsov et al. 1994; Ugarte 1995) and in the circumstellar envelopes of evolved stars and other astrophysical environments (see, e.g., Kroto et al. 1987; Kroto 1997; and Draine 2009). It is therefore reasonable to suggest that fullerenes and buckyonions form and destroy inside the SS Cyg system during quiescence, thus contributing to the observed effect of a variable reddening. It should also be possible that fullerenes and buckyonions are accreted and/or ejected during outbursts.

Indeed Iglesias-Groth (2004) discussed a model of the photoabsorption spectra of fullerenes and buckyonions in order to ascertain their presence in space by comparison with the observed features of interstellar extinction. The photoabsorption cross sections of individual and multishell fullerenes obtained by her model resemble the UV bump at $2175 \AA$ (5.7 eV). Comparison between the theoretical cross-sections and the UV bump data allows an estimate of the density of fullerenes and buckyonions in the diffuse ISM. In particular, the power law size distribution obtained can explain the positions and widths observed for the $2175 \AA$ bump. The densities inferred for small fullerenes and buckyonions is 0.2 and $0.1 \mathrm{ppm}$, respectively. Similar densities have been observed in meteorites at $\sim 0.1 \mathrm{ppm}$.

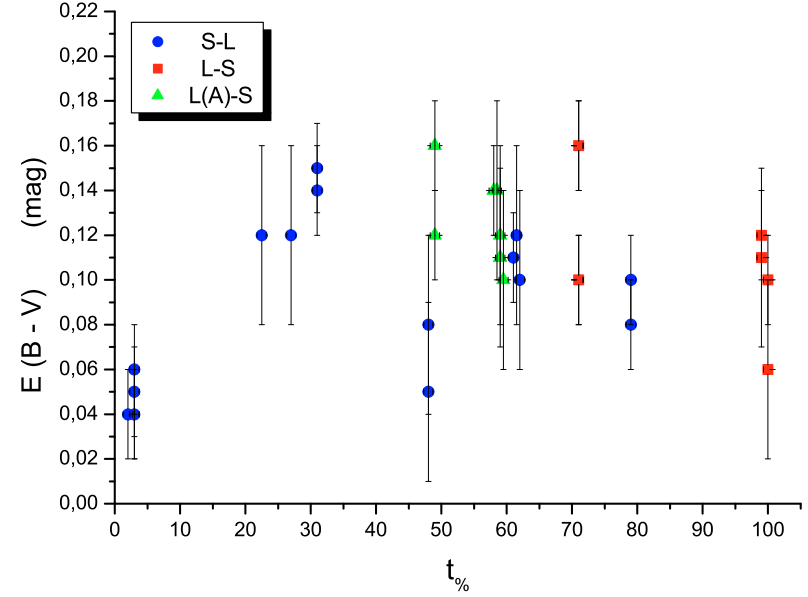

Fig. 4. $E(B-V)$ values versus $t_{\%}$ for all spectra.

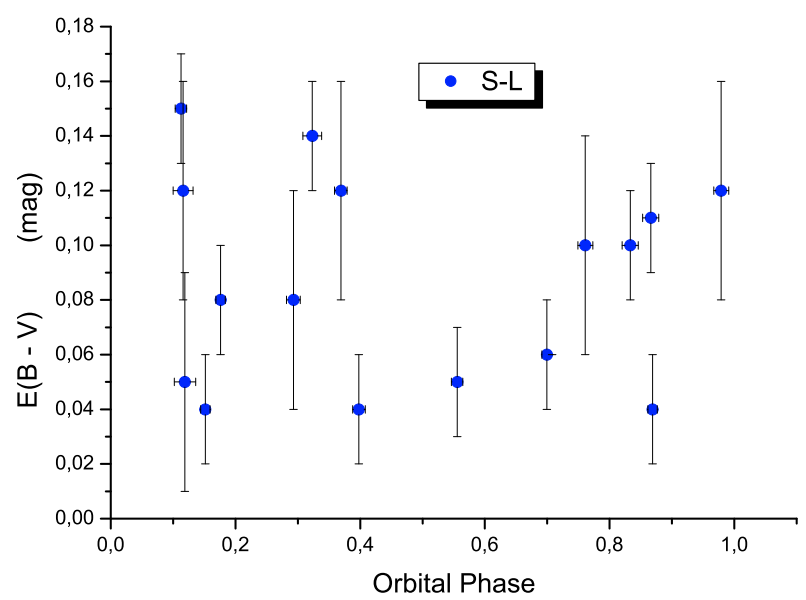

Fig. 5. $E(B-V)$ values versus the orbital phase only for the spectra belonging to a quiescence between a short and a long outburst.

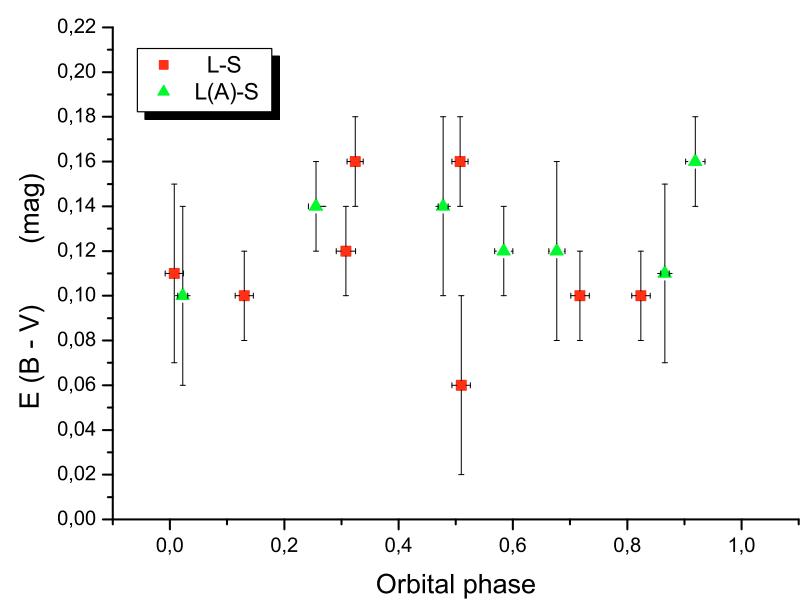

Fig. 6. $E(B-V)$ values versus the orbital phase only for the spectra belonging to a quiescence between a long/long(anomalous) and a short outburst. 


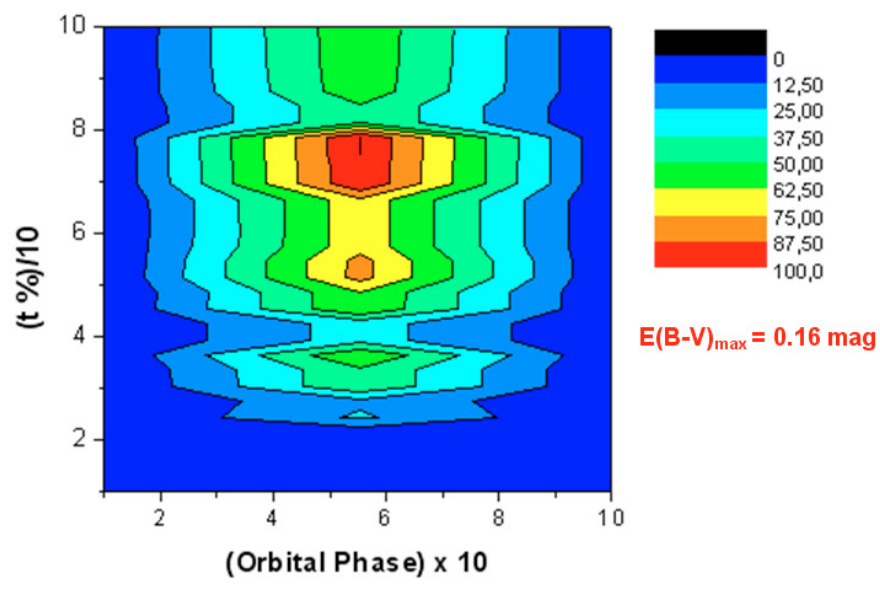

Fig. 7. $E(B-V)$ contour in the $\Phi-t \%$ plane. The maximum value $E(B-V)_{\max }=0.16$ mag corresponds to $100 \%$ of $E(B-V)$ depicted in red.

With a mixture of $\sim 80$ carbons per million hydrogen atoms, and the hypothesis that the cosmic carbon abundance is close to the solar atmosphere value, individual fullerenes may comprise $20 \%-25 \%$ of the total carbon in diffuse interstellar space.

We propose that fullerenes and buckyonions are a reasonable source of the additional, intrinsic components in SS Cygni.

Indeed, from the point of view of the physical conditions similar to those present in stellar environments, it has been shown in the laboratory that

- the grafitization and growth of fullerenes have been observed by Ugarte (1992) under strong electronic irradiation, conditions that can partially simulate those present in stellar environments;

- a strong gradient in temperature can favor the formation of fullerenes (Saidane et al. 2004);

- the formation of fullerenes has been observed under the most diverse conditions of pressure $\left(\sim 3-10^{5} \mathrm{~Pa}\right)$ (Saidane et al. 2004; Kroto et al. 1985);

- the other allotropic forms of $\mathrm{C}$ aggregate in normal conditions, for temperatures on the order of $1100-3000{ }^{\circ} \mathrm{C}$ (Ugarte 1995), and fullerenes can grow, starting on graphite and diamond seed crystals (Ugarte 1995; Tomita et al. 2004);

- in different laboratory experiments (Praburam \& Goree 1995; Saidane et al. 2004) fullerenes accreted from carbon soot for a temperature of $\sim 300-4000 \mathrm{~K}$ in vapors of inert gases (He, Ar).

From the point of view of the chemical composition, the most probable stellar candidates seem to be hydrogen-poor stars, or, better, carbon-rich systems. But these types of stars do not show a strong $2175 \AA$ peak. It has been verified in laboratory experiments that an excess of carbon or oxygen content in a gas mixture inhibits diamond formation, which can be precursors of fullerene growth (Ugarte 1995). Moreover Kroto et al. (1987) showed that in complex environments, high temperatures favor the formation of carbon/carbon bonds relative to carbon/hydrogen bonds because of the large difference in bond energies.

\subsection{Interstellar and intrinsic components of the reddening}

The results obtained from our re-analysis of the data presented here demonstrate the existence of two components of the reddening: an interstellar component that remains constant and equal to 0.04 mag and an intrinsic component, owing to concentration of matter inside the system itself. This intrinsic component is responsible for both the observed modulations with the orbital phase and for the variations during the quiescent phase. The modulation with orbital phase is caused by simply the change in orientation of the observer to the system, while the variations of the reddening with $t \%$ can be owing to a dynamic process of formation, evanescence, and escape or accretion of absorbing material.

In particular, the observed $E(B-V)$ behaviour as a function of $t_{\%}$ (see Fig. 4) indicates that immediately after the outburst the contribution of the intrinsic component is equal to zero because of a higher value of delivered radiation pressure sweeping it away. Increasing $t \%$ between 0 and $\sim 50$, the $E(B-V)$ value also increases, which indicates accreting diffuse matter inside the system. Part of this matter can convert itself in fullerenes on a time scale much shorter than the duration of the quiescence. Then, the $E(B-V)$ value decreases: this could be compatible with an escape from the system of the accumulated matter for reasons that we have discussed previously. These speculations on the escape, evanescence, or accretion of the absorbing material must be regarded as preliminary.

\section{Conclusions}

Concerning the present study on $E(B-V)$ in SS Cygni, some important considerations must be taken into account with respect to the others available in literature:

- We have utilized two independent methods and a significance test for fitting the $2175 \AA$ bump and determining the $E(B-V)$ value for each of the 31 spectral samples obtained from the IUE archive.

- Some studies (Iglesias-Groth 2004) demonstrate that fullerenes and buckyonions are the best candidates for explaining the shape, width and peak energy of the most prominent feature of the $2175 \AA$ bump.

- Other studies (Kuznetsov et al. 1994; Ugarte 1995; Kroto et al. 1987; Kroto 1997; Draine 2009) demonstrate that the formation of fullerenes and buckyonions is confirmed both in laboratory and in circumstellar envelopes of evolved stars and other astrophysical environments.

- The very high obtained significance value of $99.95 \%$, confirming the hypothesis for having $E(B-V)>0.04 \mathrm{mag}$, points out the necessity of investigating the reason of this variation taking into account the fullerenes hypothesis rather than that of the hydrogen column density.

- Indeed, following the formula of Bohlin et al. (1978) - which however has been derived for early-type stars with distances much greater than that of SS Cygni - we have derived the values of $E(B-V)$ for SS Cyg by using hydrogen column densities evaluated by SUZAKU $\left(8.2 \times 10^{19} \mathrm{~cm}^{-2}-\right.$ Ishida et al. 2009), CHANDRA $\left(5.0 \times 10^{19} \mathrm{~cm}^{-2}-\right.$ Mauche 2004), EUVE $\left(4.4 \times 10^{19} \mathrm{~cm}^{-2}-\right.$ Mauche et al. 1995), ASCA $\left(<3 \times 10^{19} \mathrm{~cm}^{-2}-\right.$ Baskill et al. 2005) which give for $E(B-V)$ the values $0.014,0.009,0.008$, and $0.005 \mathrm{mag}$, respectively. However, all these values of NH reported in the aforementioned papers are model-dependent. 
Then, our minimum value of $E(B-V)=0.04 \mathrm{mag}$ is about four times higher than the maximum reddening owing to hydrogen column density (0.014 mag). This means that the remnant reddening is caused by carbon (IglesiasGroth 2004). Our $E(B-V)$ reaches the value of 0.16 mag; this means that there is an additional local component of dust. This confirms Iglesias-Groth's hypothesis concerning the fullerenes (C) as responsible for the UV $2175 \AA$ bump.

Therefore, the reddening values obtained from our data allow us to argue that

1. the reddening of the cataclysmic variable SS Cygni, during quiescence, is variable both with the time elapsed from the preceding outburst and with the orbital phase. It consists of two components:

- the first component is interstellar in origin and is constant. The value of the correction for such a minimum reddening is $E(B-V)=0.04 \mathrm{mag}$;

- the second component is intrinsic to the system and is variable in time. The maximum value of $E(B-V)=$ $0.16 \mathrm{mag}$ is at about $70 \leq t \% \leq 80$ and $0.5 \leq \Phi \leq 0.6$ (see Fig. 7). However, note that the enhancement of $E(B-V)$ starts at $\Phi \sim 0.2$, where its value is the minimum (0.04 mag), growing until the maximum and decreasing again until it ends at $\Phi \sim 0.9$, where its value is again the minimum. And the growing of $E(B-V)$, along the quiescent phase, starts at about $t \% \sim 30$ for reaching the maximum at about 70-80 and then decreasing again until the next outburst. In other words, soon after the end of an outburst the reddening is at the minimum value; this indicates that no opaque material is present in the system, which on the contrary forms during the following days after the outburst.

2. the physical and chemical parameters of the system are consistent with the possibility of formation of fullerenes. The presence of a variable component of the reddening growing in the first half of the quiescence is compatible with the formation of fullerenes by conversion of matter inside the binary system. In the second half, the decreasing value of $E(B-V)$ could be compatible with an escape from the system of the accumulated matter.

We therefore believe that we have answered the question that triggered this paper: why are the values of $E(B-V)$ available in the literature for SS Cyg so different? Indeed, two components of the reddening of SS Cyg emerge from our analysis: the first is interstellar in origin, and the second (intrinsic to the system itself) is variable and changes during the evolution of a quiescent phase. Moreover, an orbital modulation also exists.

A possible model explaining the modulations owing to orbital phase is beyond the scope of this paper.

More investigations in other CVs are desirable in order to test the general validity of our results. Measurements in sub-mm and $\mathrm{mm}$ bands are crucial for such a test. The ALMA groundbased experiment $(0.3-16.7 \mathrm{~mm})$ (see Wootten 2008) and the future space-based experiment, MILLIMETRON $(0.02-20 \mathrm{~mm})$ (Kardashev \& Novikov 2010) will help to determine the question raised here about the extent of the presence of dust in CVs.

Acknowledgements. Many thanks to Corinne Rossi of "La Sapienza" University in Roma for helpful discussions. We are grateful to the unknown referee for his important suggestions, which allowed us to improve the paper. We express our thanks to Jim Beall of St. John's College and the Space Sciences Division of the Naval Research Laboratory for his assistance with a final draft of this paper. The authors MM, MC, and RL are grateful to the first author, Silvia Gaudenzi, for having worked - with FG's help - on the revision of this paper in spite of her serious health problems. This research has made use of NASA's Astrophysics Data System.

\section{References}

Baskill, D. S., Wheatley, P. J., \& Osborne, J. P. 2005, MNRAS, 357, 626

Bath, G. T., \& van Paradijs, J. 1983, Nature, 305, 33

Bohlin, R. C., Savage, B. D., \& Drake, J. F. 1978, ApJ, 224, 132

Bruch, A., \& Engel, A. 1994, A\&AS, 104, 79

Cordova, F. A., \& Mason, K. O. 1983, in Accretion Driven Stellar X-Rays Sources, ed. W. H. G. Lewin, \& E. D. J. van den Heuvel (Cambridge: Cambridge University Press), 147

Draine, B. T. 2009, in Cosmic Dust - Near and Far, ed. T. Henning, E. Grün, \& J. Steinacker, ASP Conf. Ser., 414, 453

Dubus, G., Campbell, R., Kern, B., Taam, R. E., \& Spruit, H. C. 2004, MNRAS, 349,869

Fabbiano, G., Hartmann, L., Raymond, J., et al. 1981, ApJ, 243, 911

Feldt, A. N., \& Chincarini, G. 1980, PASP, 92, 528

Fitzpatrick, E. L., \& Massa, D. 1986, ApJ, 307, 286

Fitzpatrick, E. L., \& Massa, D. 1988, ApJ, 328, 734

Gaudenzi, S., Claudi, R. U., Giovannelli, F., et al. 2002, in Multifrequency Behaviour of High Energy Cosmic Sources, ed. F. Giovannelli, \& L. SabauGraziati, Mem. SAIt., 73, 213

Giovannelli, F. 2008, ChJA\&A Suppl., 8, 237

Giovannelli, F., \& Martinez-Pais, I. G. 1991, SSRv, 56, 313

Giovannelli, F., Gaudenzi, S., Rossi, C., \& Piccioni, A. 1983, AcA, 33, 319

Giovannelli, F., Bartolini, C., Candy, P., et al. 1984, in Proc. of the Fourth European IUE Conf., ed. E. Rolfe, ESA SP, 218, 391

Giovannelli, F., Gaudenzi, S., Lombardi, R., et al. 1985, in Multifrequency Behaviour of Galactic Accreting Sources, ed. F. Giovannelli, SIDEREA, Roma, Italy, 37

Gnedin, Yu. N., Natsvlishvili, T. M., Shtol', V. G., Valyavin, G. G., \& Shakhovskoi, N. M. 1995, Sov. AstL, 21, 118

Hack, M., \& La Dous, C. 1990, Cataclysmic Variables and Related Objects, NASA/CNRS Monograph Series on Non Thermal Phenomena in Stellar Atmospheres

Hack, M., \& La Dous, C. 1993, Cataclysmic Variables and Related Objects, ASSL, 101, 1

Harrison, T. E., McNamara, B. J., Szkody, P., et al. 1999, ApJ, 515, L93

Hellier, C. 2001, Cataclysmic Variable Stars (Springer)

Holm, A. V., \& Gallagher, J. S. 1974, ApJ, 192, 425

Howarth, I. D. 1978, JBAS, 88, 458

Iglesias-Groth, S. 2004, ApJ, 608, L37

Ishida, M., Okada, S., Hayashi, T., Nakamura, R., \& Terada, Y. 2009, PASJ, 61, S77

Jameson, R. F., King, A. R., Bode, M. F., \& Evans, A. 1987, The Observatory, 107,72

Kardashev, N. S., \& Novikov, I. D. 2010, talk at the I.M.C. Workshop 2010: Science with Millimetron, Palermo, Italy, 14th-18th June 2010

Kiplinger, A. L. 1979, AJ, 84, 655

Kjurkchieva, D., Marchev, D., \& Ogloza, W. 1998, Ap\&SS, 262, 53

Körding, E., Rupen, M., Knigge, C., Fender, R., \& Dhawan, V. 2008, Science, 320,1318

Kroto, H. 1997, RvMP, 69, 703

Kroto, H. W., Heath, J. R., O'Brien, S. C., Curl, R. F., \& Smaey, R. E. 1985, Nature, 318, 162

Kroto, H. W., Heath, J. R., O’Brien, S. C., Curl, R. F., \& Smaey, R. E. 1987, ApJ, 314,352

Kuznetsov, V. L., Chuvilin, A. L., Butenko, Y. V., Mal'kov, I. Y., \& Titov, V. M. 1994, Chem. Phys. Lett., 222, 343

La Dous, C. 1991, A\&A, 252, 100

La Dous, C. 1994, SSRv, 67, 1

Landi, R., Bassani, L., Dean, J., Bird, J., \& Fiocchi, M. 2009, MNRAS, 392, 630

Livio, M., Shaviv, G. 1984, Cataclysmic Variables and Related Objects, Haifa, Israel, August 9-13, 1982, Association of Univ. for Research in Astronomy, IAU Coll., 072

Long, K. S., Froning, C. S., Knigge, C., et al. 2005, ApJ, 630, 511

Marchev, D., Kjurkchieva, D., \& Ogłoza, W. 1999, AcA, 49, 585

Martinez-Pais, I. G., Giovannelli, F., Rossi, C., \& Gaudenzi, S. 1994, A\&A, 291, 455

Mattei, J. A., Saladyga, M., \& Waagen, E. O. 1985, AAVSO Monograph (Cambridge, MA, USA: American Association of Variable Star Observers (AAVSO))

Mattei, J. A., Waagen, E. O., \& Foster, E. G. 1991, AAVSO Monogr. (Cambridge, MA, USA: American Association of Variable Star Observers (AAVSO)), 1

Mauche, C. W. 1996, ApJ, 463, L87 
A\&A 525, A147 (2011)

Mauche, C. W. 2004, ApJ, 610, 422

Mauche, C. W., Raymond, J. C., \& Mattei, J. A. 1995, ApJ, 446, 842

Mauche, C. W., Lee, Y. P., \& Kallman, T. R. 1997, ApJ. 477, 832

Mitrofanov, I. 1978, Sov. AstL, 4, 119

NED Catalog: http://nedwww . ipac. caltech. edu/

Okada, S., Nakamura, R., \& Ishida, M. 2008, ApJ, 680, 695

Patterson, J. 1984, ApJS, 54, 443

Patterson, J. 1994, PASP, 106, 209

Patterson, J. 1998, PASP, 110, 1132

Praburam, G., \& Goree, J. 1995, ApJ, 441, 830

Ricketts, M. J., King, A. R., \& Raine, D. J. 1979, MNRAS, 186, 233

Ritter, H. 2008 [arXiv:astro-ph/0809. 1800v1]

Robinson, E. L. 1976, ARA\&A, 14, 119

Saidane, K., Razafinimanana, M., Lange, H., et al. 2004, J. Phys. D: Appl. Phys., 37,232

Scaringi, S., Bird, A. J., Norton, A. J., Knigge, C., \& Hill, A. B. 2010, MNRAS 401, 2207

Schlegel, D. J., Finkbeiner, D. P., \& Davis, M. 1998, ApJ, 500, 525

Schreiber, M. R., Hameury, J.-M., \& Lasota, J.-P. 2003, A\&A, 410, 239
Schreiber, M. R., \& Lasota, J.-P. 2007, A\&A, 473, 897

Seaton, M. J. 1979, MNRAS, 187, 73

Smak, J. 1985a, in Multifrequency Behaviour of Galactic Accreting Sources, ed. F. Giovannelli, SIDEREA, Roma, Italy, 17

Smak, J. 1985b, in Multifrequency Behaviour of Galactic Accreting Sources, ed. F. Giovannelli, SIDEREA, Roma, Italy, 3

Szkody, P. 1977, ApJ, 217, 140

Tomita, S., Fujii, M., \& Hayashi, S. 2004, ApJ, 609, 220

Ugarte, D. 1992, Nature, 359, 707

Ugarte, D. 1995, ApJ, 443, L85

Verbunt, F. 1987, A\&AS, 71, 339

Wallerstein, G. 1963, ApJ, 137, 991

Warner, B. 1995, Cataclysmic Variable Stars, Cambridge Astrophysics Series (Cambridge University Press)

Wootten, A. 2008, ApSS, 313, 9

Wu, C.-C. 1976, in Structure and Evolution of Close Binary Systems, ed. P. Eggleton, S. Mitton, \& J. Whelan (Dordrecht: D. Reidel Publishing Co.), IAU Symp., 73, 213

Zuckerman, M.-C. 1961, Ann. Astrophys., 6, 431 
Table 1. Journal of observations.

\begin{tabular}{|c|c|c|c|c|c|c|}
\hline$n$ & Spectrum & $\begin{array}{c}\text { HJD } \\
2440000+\end{array}$ & $\phi \pm \sigma_{\phi}$ & $\begin{array}{c}t_{\mathrm{exp}} \\
(\mathrm{mm} \mathrm{ss})\end{array}$ & $\begin{array}{c}t \% \\
( \pm 1)\end{array}$ & $n_{x}$ \\
\hline 1 & SWP03880 & 3884.92029 & $0.364 \pm 0.012$ & 1600 & 2 & $1 \mathrm{a}$ \\
\hline 2 & LWR03452 & 3884.93928 & $0.432 \pm 0.018$ & 2500 & 2 & 1a \\
\hline 3 & SWP03881 & 3884.96281 & $0.517 \pm 0.012$ & 1600 & 3 & $1 \mathrm{a}$ \\
\hline 4 & LWR03453 & 3884.98396 & $0.594 \pm 0.014$ & 1900 & 3 & $1 \mathrm{a}$ \\
\hline 5 & SWP03882 & 3885.00266 & $0.662 \pm 0.012$ & 1600 & 3 & $1 \mathrm{a}$ \\
\hline 6 & LWR03454 & 3885.02356 & $0.737 \pm 0.011$ & 1500 & 3 & $1 \mathrm{a}$ \\
\hline 7 & SWP03883 & 3885.04606 & $0.819 \pm 0.012$ & 1600 & 3 & $1 \mathrm{a}$ \\
\hline 8 & LWR03455 & 3885.07370 & $0.9190 \pm 0.0087$ & 1200 & 3 & $1 \mathrm{a}$ \\
\hline 9 & SWP03884 & 3885.10318 & $0.026 \pm 0.029$ & 4000 & 3 & $1 \mathrm{a}$ \\
\hline 10 & LWR03456 & 3885.12731 & $0.1130 \pm 0.0087$ & 1200 & 3 & $1 \mathrm{a}$ \\
\hline 11 & SWP03885 & 3885.14848 & $0.190 \pm 0.012$ & 1600 & 3 & $1 \mathrm{a}$ \\
\hline 12 & SWP03939 & 3892.13919 & $0.4990 \pm 0.0065$ & 0900 & 24 & $1 \mathrm{a}$ \\
\hline 13 & SWP03952 & 3892.92250 & $0.335 \pm 0.015$ & 2000 & 27 & $1 \mathrm{a}$ \\
\hline 14 & LWR03524 & 3892.94147 & $0.403 \pm 0.015$ & 2000 & 27 & $1 \mathrm{a}$ \\
\hline 15 & LWR04087 & 3954.60421 & $0.6480 \pm 0.0073$ & 1000 & 38 & $1 b$ \\
\hline 16 & SWP04722 & 3954.62921 & $0.738 \pm 0.039$ & 54 & 3 & $1 b$ \\
\hline 17 & SWP05263 & 4010 & 0.36 & & 20 & $1 \mathrm{c}$ \\
\hline 18 & SWP05264 & 4010.9 & $0.479 \pm 0.015$ & 2100 & 20 & $1 \mathrm{c}$ \\
\hline 19 & SWP05265 & 4010.93836 & $0.600 \pm 0.017$ & 2400 & 20 & $1 \mathrm{c}$ \\
\hline 20 & SWP05266 & 4010.97185 & $0.721 \pm 0.017$ & 2400 & 20 & $1 \mathrm{c}$ \\
\hline 21 & SWP05267 & 4011.00557 & $0.844 \pm 0.017$ & 2400 & 20 & $1 \mathrm{c}$ \\
\hline 22 & SWP05268 & 4011.03919 & $0.965 \pm 0.017$ & 2400 & 20 & $1 \mathrm{c}$ \\
\hline 23 & SWP05269 & 4011.07320 & $0.088 \pm 0.017$ & 2400 & 20 & $1 \mathrm{c}$ \\
\hline 24 & SWP05270 & 4011.10699 & $0.211 \pm 0.017$ & 2400 & 20 & $1 \mathrm{c}$ \\
\hline 25 & SWP05271 & 4011.14089 & $0.333 \pm 0.017$ & 2400 & 20 & $1 \mathrm{c}$ \\
\hline 26 & LWR13585 & 5152.54065 & $0.718 \pm 0.015$ & 2000 & 22 & $2 \mathrm{a}$ \\
\hline 27 & SWP17338 & 5152.57117 & $0.829 \pm 0.037$ & 5100 & 22 & $2 \mathrm{a}$ \\
\hline 28 & LWR13586 & 5152.60128 & $0.938 \pm 0.018$ & 2500 & 22 & $2 \mathrm{a}$ \\
\hline 29 & SWP17339 & 5152.62364 & $0.019 \pm 0.015$ & 2000 & 23 & $2 \mathrm{a}$ \\
\hline 30 & LWR13587 & 853 & $0.109 \pm 0.017$ & 00 & 23 & $2 \mathrm{a}$ \\
\hline 31 & LWR13637 & 515 & 0.07 & 0 & 48 & $2 \mathrm{a}$ \\
\hline 32 & SWP & 515 & 0.16 & 40 & 48 & $2 \mathrm{a}$ \\
\hline 33 & LWR13638 & 5159.5 & $0.250 \pm 0.013$ & 1800 & 48 & $2 \mathrm{a}$ \\
\hline 34 & SWP17388 & 5159.61653 & $0.336 \pm 0.018$ & 2500 & 48 & $2 \mathrm{a}$ \\
\hline 35 & LWR13639 & 5159.64186 & $0.428 \pm 0.012$ & 1700 & 48 & $2 \mathrm{a}$ \\
\hline 36 & SWP17414 & 5163.58971 & $0.721 \pm 0.018$ & 2500 & 62 & $2 \mathrm{a}$ \\
\hline 37 & LWR13668 & 5163.61183 & $0.801 \pm 0.015$ & 2000 & 62 & $2 \mathrm{a}$ \\
\hline 38 & SWP17415 & 5163.63683 & $0.892 \pm 0.029$ & 4000 & 63 & $2 \mathrm{a}$ \\
\hline 39 & SWP23828 & 5943.95030 & $0.036 \pm 0.015$ & 2000 & 62 & $3 \mathrm{a}$ \\
\hline 40 & LWP04114 & 5943.96775 & $0.099 \pm 0.012$ & 1600 & 62 & $3 a$ \\
\hline 41 & SWP23829 & 5943.98785 & $0.172 \pm 0.011$ & 1500 & 62 & $3 \mathrm{a}$ \\
\hline 42 & LWP04115 & 5944.00808 & $0.2450 \pm 0.0036$ & 0500 & 62 & $3 a$ \\
\hline 43 & LWP04116 & 5944.06430 & $0.4480 \pm 0.0036$ & 0500 & 62 & $3 \mathrm{a}$ \\
\hline 44 & LWP04862 & 6026.68288 & $0.574 \pm 0.012$ & 1600 & 51 & $3 b$ \\
\hline 45 & SWP24533 & 6026.70570 & $0.656 \pm 0.016$ & 0 & 51 & $3 b$ \\
\hline 46 & LW & 602 & 0.72 & 12 & 52 & $3 b$ \\
\hline 47 & SWP29929 & 6786.09239 & $0.082 \pm 0.015$ & 2000 & 31 & $4 a$ \\
\hline 48 & LWP09743 & 6786.10925 & $0.143 \pm 0.011$ & 1500 & 31 & $4 a$ \\
\hline 49 & SWP29930 & 6786.14755 & $0.281 \pm 0.029$ & 4000 & 31 & $4 a$ \\
\hline 50 & LWP09744 & 6786.17074 & $0.3650 \pm 0.0094$ & 1300 & 31 & $4 a$ \\
\hline 51 & SWP29931 & 6786.19457 & $0.452 \pm 0.012$ & 1600 & 31 & $4 a$ \\
\hline 52 & LWP09851 & 6799.83248 & $0.8290 \pm 0.0073$ & 1000 & 61 & $4 a$ \\
\hline 53 & SWP30016 & 6799.85269 & $0.902 \pm 0.026$ & 3600 & 61 & $4 a$ \\
\hline 54 & LWP09852 & 6799.89936 & $0.071 \pm 0.015$ & 2000 & 61 & $4 a$ \\
\hline 55 & SWP30017 & 6799.92385 & $0.160 \pm 0.029$ & 4000 & 62 & $4 a$ \\
\hline 56 & LWP09853 & 6799.96350 & $0.304 \pm 0.012$ & 1600 & 62 & $4 a$ \\
\hline 57 & LWP09910 & 6807.83190 & $0.7920 \pm 0.0087$ & 1200 & 79 & $4 a$ \\
\hline 58 & SWP30078 & 6807.85444 & $0.874 \pm 0.025$ & 3500 & 79 & $4 a$ \\
\hline 59 & LWP09911 & 6807.87640 & $0.953 \pm 0.010$ & 1400 & 79 & $4 \mathrm{a}$ \\
\hline 60 & SWP30079 & 6807.90531 & $0.058 \pm 0.025$ & 3500 & 79 & $4 \mathrm{a}$ \\
\hline
\end{tabular}


Table 1. continued.

\begin{tabular}{|c|c|c|c|c|c|c|}
\hline$n$ & rum & $\begin{array}{c}\text { HJD } \\
2440000+\end{array}$ & $\sigma_{\phi}$ & $\begin{array}{c}t_{\exp } \\
\text { (mm ss) }\end{array}$ & $\begin{array}{c}t \% \\
( \pm 1)\end{array}$ & $n_{x}$ \\
\hline 61 & LWP09912 & 6807.92700 & $0.136 \pm 0.010$ & 1400 & 79 & $4 \mathrm{a}$ \\
\hline 62 & SWP30080 & 307.94888 & $0.216=$ & 1500 & 79 & $4 a$ \\
\hline 63 & SWP30135 & 6816.98833 & $0.944 \pm 0.029$ & 4000 & 99 & $4 a$ \\
\hline 64 & LWP09977 & 6817.01377 & $0.036 \pm 0.012$ & 1600 & 99 & $4 a$ \\
\hline 65 & LWP09978 & 6817.06612 & $0.226 \pm 0.011$ & 1500 & 99 & $4 a$ \\
\hline 66 & SWP30136 & 6817.08398 & $0.290 \pm$ & 4000 & 99 & $4 a$ \\
\hline 67 & SWP & & 0.34 & & 99 & $4 a$ \\
\hline 68 & LWP11235 & 6994.52059 & 0.72 & 1500 & 23 & $5 \mathrm{a}$ \\
\hline 69 & SWP31355 & 4617 & $0.814 \pm 0.029$ & 4000 & 23 & 59 \\
\hline 70 & LWP11236 & 6994.57002 & $0.900 \pm 0.011$ & 1500 & 23 & 59 \\
\hline 71 & SWP31356 & 6994.60307 & 0.020 & 4300 & 23 & 59 \\
\hline 72 & LWP16969 & 7881.94325 & $0.779 \pm$ & 1600 & 71 & 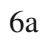 \\
\hline 73 & SWP37866 & 7881.96804 & $0.869 \pm 0.029$ & 4000 & 71 & $=$ \\
\hline 74 & LWP16970 & 7881.99276 & $0.958 \pm 0.012$ & 1600 & 71 & $\mathrm{a}$ \\
\hline 75 & SWP37867 & 7882.02741 & $0.084=$ & 4000 & 71 & $6 a$ \\
\hline 76 & LWP16971 & 7882.05298 & $0.176 \pm 0.012$ & 1600 & 71 & $6 a$ \\
\hline 77 & SWP37868 & 7882.08221 & $0.282 \pm 0.025$ & 3500 & 71 & $6 a$ \\
\hline 78 & LWP16972 & 788 & $0.366 \pm$ & 1600 & 71 & $6 a$ \\
\hline 79 & SW & & & & 71 & $6 a$ \\
\hline 80 & LW & & & & 71 & $6 a$ \\
\hline 81 & & & & & 71 & $6 a$ \\
\hline 82 & LWF & & 0.9 & 16 & & $6 a$ \\
\hline 83 & SWF & & 0.0 & 4000 & 9 & $6 a$ \\
\hline 84 & LWI & & 0.1 & 18 & 9 & $6 a$ \\
\hline 85 & SWP37925 & 789 & 0.260 & 4200 & 99 & $\mathrm{a}$ \\
\hline 86 & LWP17054 & 789 & 0.356 & 1800 & 99 & $a$ \\
\hline 87 & SWP37926 & & $0.463=$ & 4000 & 100 & 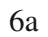 \\
\hline 88 & LWP17055 & .10056 & 0.556 & 16 & 100 & $6 a$ \\
\hline 89 & SWP37927 & 7892.13210 & 0.670 & 4000 & 100 & $6 a$ \\
\hline 90 & LWP17056 & 7892.15841 & $0.765 \pm 0$ & 1600 & 100 & $6 a$ \\
\hline 91 & SWP39027 & 8050.07618 & 0.5 & 4000 & 49 & $7 \mathrm{a}$ \\
\hline 92 & LWP & 164 & & & 49 & $7 \mathrm{a}$ \\
\hline 93 & SWP & 8050.1 & 0.7 & & 49 & $7 \mathrm{a}$ \\
\hline 94 & LWI & & 036 & & 49 & $7 \mathrm{a}$ \\
\hline 95 & & & & & 4 & $7 \mathrm{a}$ \\
\hline 96 & LV & 80 & 094 & & 5 & $7 \mathrm{a}$ \\
\hline 97 & SW & & 25 & 0 & 5 & $7 \mathrm{a}$ \\
\hline 98 & LWP & & 0.29 & 0700 & 58 & $7 \mathrm{a}$ \\
\hline 99 & SWP & 17 & 0.43 & 2000 & 58 & $7 \mathrm{a}$ \\
\hline 100 & LWP & 5869 & 0.5260 & 1200 & 59 & $7 \mathrm{a}$ \\
\hline 101 & SWP39050 & 8052.58901 & 0.636 & 3000 & 59 & $7 \mathrm{a}$ \\
\hline 102 & LWP18074 & & 0.0080 & 1100 & 5 & $7 \mathrm{a}$ \\
\hline 103 & SWP39051 & 8052.63858 & 0.012 & 1700 & 59 & $7 \mathrm{a}$ \\
\hline 104 & LWP18075 & 8052.66629 & $0.9160 \pm 0.0080$ & 1100 & 59 & $7 \mathrm{a}$ \\
\hline 105 & SWP39052 & 8052.69497 & $0.020 \pm 0.014$ & 1900 & 59 & $7 \mathrm{a}$ \\
\hline 106 & & & & 1500 & 60 & $7 \mathrm{a}$ \\
\hline 107 & & & & & 11 & $7 \mathrm{~b}$ \\
\hline 108 & & & & & 27 & $7 \mathrm{~b}$ \\
\hline 109 & & & & & 27 & $7 b$ \\
\hline 110 & & & & $60 \Omega 0$ & 27 & $7 \mathrm{~b}$ \\
\hline 111 & LWP19070 & & & 17 & 27 & $7 b$ \\
\hline 112 & SWP46280 & & $0.378=$ & 3000 & 56 & (2) \\
\hline 113 & SWP46281 & 8942.71350 & $0.545 \pm 0$ & 3000 & 56 & 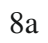 \\
\hline 114 & SWP46286 & & $0.016 \pm 0$ & 3000 & 57 & \\
\hline 115 & SWP46287 & 8943.72417 & $0.205 \pm$ & 4500 & 57 & 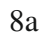 \\
\hline 116 & P46292 & 68609 & 0.688 & 30 & 59 & $8 a$ \\
\hline 117 & SWP46293 & 8944.73742 & $0.873 \pm 0.022$ & 3000 & 59 & $8 \mathrm{a}$ \\
\hline 118 & SWP51437 & 9548.17670 & $0.803 \pm 0.015$ & 2000 & 1 & $9 \mathrm{a}$ \\
\hline 119 & LWP28637 & 9548.19669 & $0.8750 \pm 0.0087$ & 1200 & 1 & $9 \mathrm{a}$ \\
\hline 120 & SWP51438 & 9548.22797 & $0.989 \pm 0.022$ & 3000 & 1 & $9 \mathrm{a}$ \\
\hline 121 & SWP51439 & 9548.26959 & $0.139 \pm 0.033$ & 4500 & 1 & \\
\hline
\end{tabular}

Notes. Columns: 1 - progressive number; 2 - IUE image number; 3 - Julian Date related to the time at the half of acquisition of spectra; 4 - orbital phase of the system calculated at half of the acquisition of the spectrum; 5 - exposure time; 6 - time elapsed since the end of the preceding outburst expressed in percent of the duration of the quiescent phase; 7 - number $\left(n_{x}, x=a, b, c\right)$ corresponding to the quiescence during which spectra were obtained. 
Table 2. Results.

\begin{tabular}{|c|c|c|c|c|c|c|}
\hline $\begin{array}{l}\text { Short and long wavelength } \\
\text { contiguous spectra }\end{array}$ & $\begin{array}{l}\text { Unified } \\
\text { spectrum }\end{array}$ & $\bar{\phi} \pm \sigma_{\bar{\phi}}$ & $\overline{\mathrm{t}}_{\%} \pm \sigma_{\overline{\mathrm{t}}_{\%}}$ & $\begin{array}{c}E(B-V) \pm \\
\Delta E(B-V) \\
\quad(\mathrm{mag})\end{array}$ & OB1 & OB2 \\
\hline SWP03880-LWR03452 & LS5280 & $0.398 \pm 0.010$ & $2.0 \pm 0.7$ & $0.04 \pm 0.02$ & $\mathrm{~S}$ & $\mathrm{~L}$ \\
\hline SWP03881-LWR03453 & LS5381 & $0.5555 \pm 0.0092$ & $3.0 \pm 0.7$ & $0.05 \pm 0.02$ & & \\
\hline SWP03882-LWR03454 & LS5482 & $0.6995 \pm 0.0081$ & $3.0 \pm 0.7$ & $0.06 \pm 0.02$ & & \\
\hline SWP03883-LWR03455 & LS5583 & $0.8690 \pm 0.0075$ & $3.0 \pm 0.7$ & $0.04 \pm 0.02$ & & \\
\hline SWP03885-LWR03456 & LS5685 & $0.1515 \pm 0.0075$ & $3.0 \pm 0.7$ & $0.04 \pm 0.02$ & & \\
\hline SWP03952-LWR03524 & LS2452 & $0.369 \pm 0.010$ & $27.0 \pm 0.7$ & $0.12 \pm 0.04$ & & \\
\hline SWP17339-LWR13586 & LS8639 & $0.979 \pm 0.012$ & $22.5 \pm 0.7$ & $0.12 \pm 0.04$ & $\mathrm{~S}$ & $\mathrm{~L}$ \\
\hline SWP17387-LWR13637 & LS3787 & $0.119 \pm 0.017$ & $48.0 \pm 0.7$ & $0.05 \pm 0.04$ & & \\
\hline SWP17388-LWR13638 & LS3888 & $0.293 \pm 0.011$ & $48.0 \pm 0.7$ & $0.08 \pm 0.04$ & & \\
\hline SWP17414-LWR13668 & LS6814 & $0.761 \pm 0.012$ & $62.0 \pm 0.7$ & $0.10 \pm 0.04$ & & \\
\hline SWP29929-LWP09743 & LS4329 & $0.1125 \pm 0.0092$ & $31.0 \pm 0.7$ & $0.15 \pm 0.02$ & $\mathrm{~S}$ & $\mathrm{~L}$ \\
\hline SWP29930-LWP09744 & LS4430 & $0.323 \pm 0.015$ & $31.0 \pm 0.7$ & $0.14 \pm 0.02$ & & \\
\hline SWP30016-LWP09851 & LS5116 & $0.866 \pm 0.013$ & $61.0 \pm 0.7$ & $0.11 \pm 0.02$ & & \\
\hline SWP30017-LWP09852 & LS5217 & $0.116 \pm 0.016$ & $61.5 \pm 0.7$ & $0.12 \pm 0.04$ & & \\
\hline SWP30078-LWP09910 & LS1078 & $0.833 \pm 0.013$ & $79.0 \pm 0.7$ & $0.10 \pm 0.02$ & & \\
\hline SWP30080-LWP09912 & LS1280 & $0.1760 \pm 0.0075$ & $79.0 \pm 0.7$ & $0.08 \pm 0.02$ & & \\
\hline SWP37866-LWP16969 & LS6966 & $0.824 \pm 0.016$ & $71.0 \pm 0.7$ & $0.10 \pm 0.02$ & $\mathrm{~L}$ & $\mathrm{~S}$ \\
\hline SWP37867-LWP16971 & LS7167 & $0.130 \pm 0.016$ & $71.0 \pm 0.7$ & $0.10 \pm 0.02$ & & \\
\hline SWP37868-LWP16972 & LS7268 & $0.324 \pm 0.014$ & $71.0 \pm 0.7$ & $0.16 \pm 0.02$ & & \\
\hline SWP37869-LWP16973 & LS7369 & $0.508 \pm 0.014$ & $71.0 \pm 0.7$ & $0.16 \pm 0.02$ & & \\
\hline SWP37924-LWP17052 & LS5224 & $0.008 \pm 0.016$ & $99.0 \pm 0.7$ & $0.11 \pm 0.04$ & & \\
\hline SWP37925-LWP17054 & LS5425 & $0.308 \pm 0.017$ & $99.0 \pm 0.7$ & $0.12 \pm 0.02$ & & \\
\hline SWP37926-LWP17055 & LS5526 & $0.510 \pm 0.016$ & $100.0 \pm 0.7$ & $0.06 \pm 0.04$ & & \\
\hline SWP37927-LWP17056 & LS5627 & $0.718 \pm 0.016$ & $100.0 \pm 0.7$ & $0.10 \pm 0.02$ & & \\
\hline SWP39027-LWP18050 & LS5027 & $0.584 \pm 0.016$ & $49.0 \pm 0.7$ & $0.12 \pm 0.02$ & $\mathrm{~L}(\mathrm{~A})$ & $\mathrm{S}$ \\
\hline SWP39029-LWP18051 & LS5129 & $0.919 \pm 0.017$ & $49.0 \pm 0.7$ & $0.16 \pm 0.02$ & & \\
\hline SWP39048-LWP18072 & LS7248 & $0.256 \pm 0.013$ & $58.0 \pm 0.7$ & $0.14 \pm 0.02$ & & \\
\hline SWP39049-LWP18073 & LS7349 & $0.4780 \pm 0.0087$ & $58.5 \pm 0.7$ & $0.14 \pm 0.04$ & & \\
\hline SWP39050-LWP18074 & LS7450 & $0.677 \pm 0.014$ & $59.0 \pm 0.7$ & $0.12 \pm 0.04$ & & \\
\hline SWP39051-LWP18075 & LS7551 & $0.8660 \pm 0.0075$ & $59.0 \pm 0.7$ & $0.11 \pm 0.04$ & & \\
\hline SWP39052-LWP18077 & LS7752 & $0.0225 \pm 0.0087$ & $59.5 \pm 0.7$ & $0.10 \pm 0.04$ & & \\
\hline
\end{tabular}

Notes. Columns one to seven report the spectra to be coupled; the name of the unified spectrum (it is compound by L (long) and S (short) followed by the last couple of numbers characterizing the IUE long and short wavelength spectrum, respectively); the average of orbital phase; the mean $t_{\%}$; the $E(B-V)$ value; the type of the preceding outburst $(\mathrm{OB} 1)$; the type of the following outburst $(\mathrm{OB} 2)(\mathrm{S}=\mathrm{short}, \mathrm{L}=$ long, $\mathrm{L}(\mathrm{A})=$ long(anomalous)). 